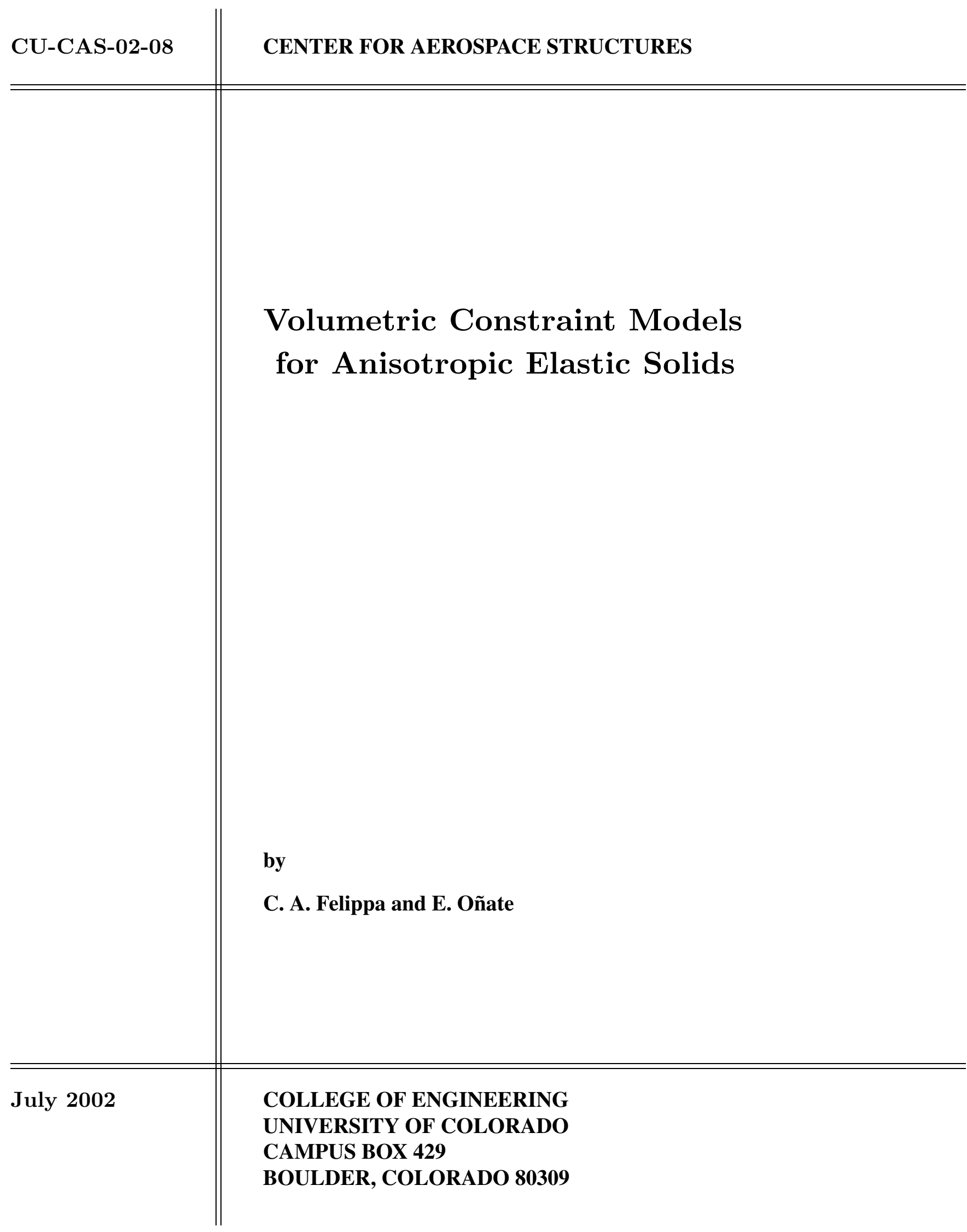




\section{Volumetric Constraint Models for Anisotropic Elastic Solids}

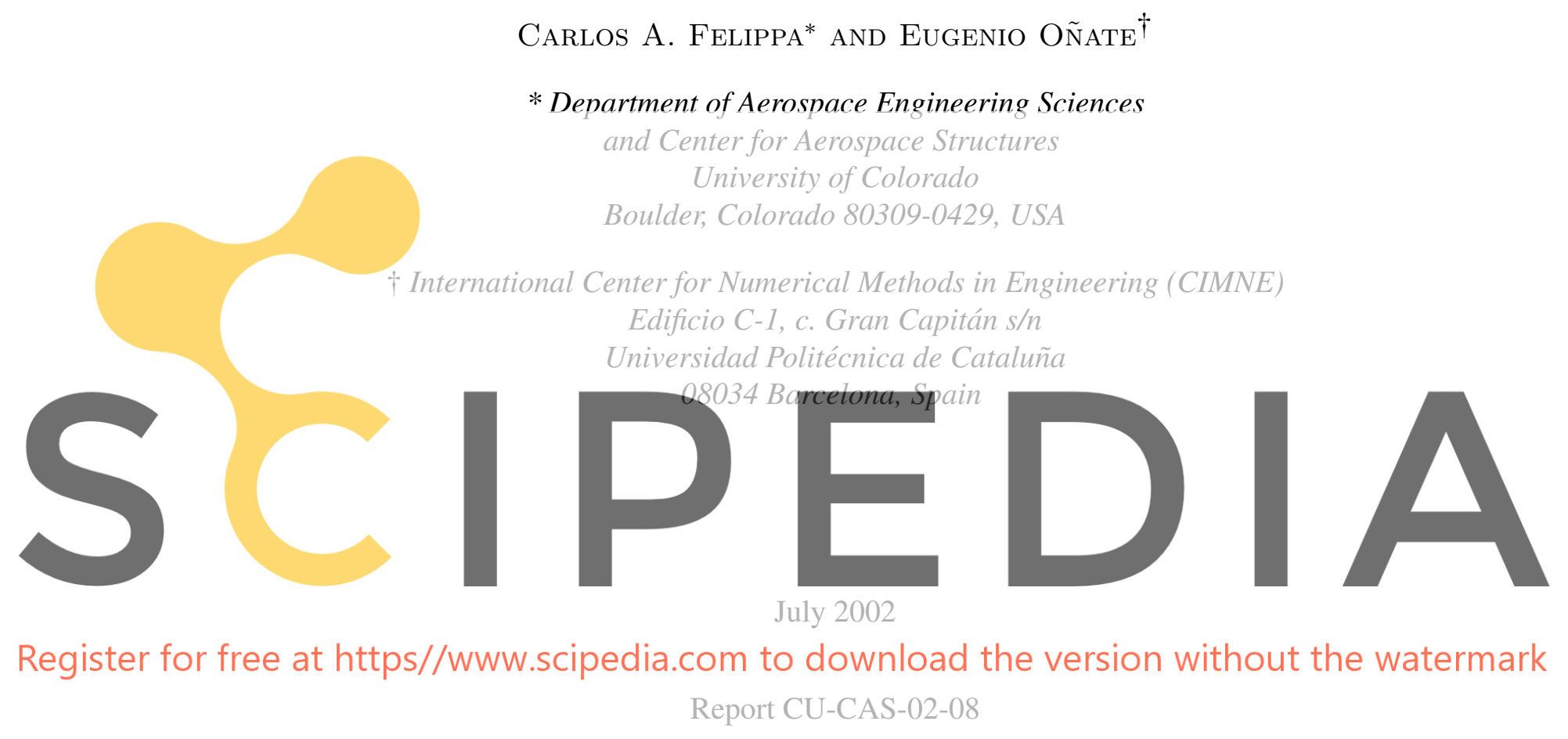

The work of the first author has been supported by the Spanish Ministerio of Educación y Cultura through a faculty fellowship while visiting CIMNE on April through July 2002. 


\section{TABLE OF CONTENTS}

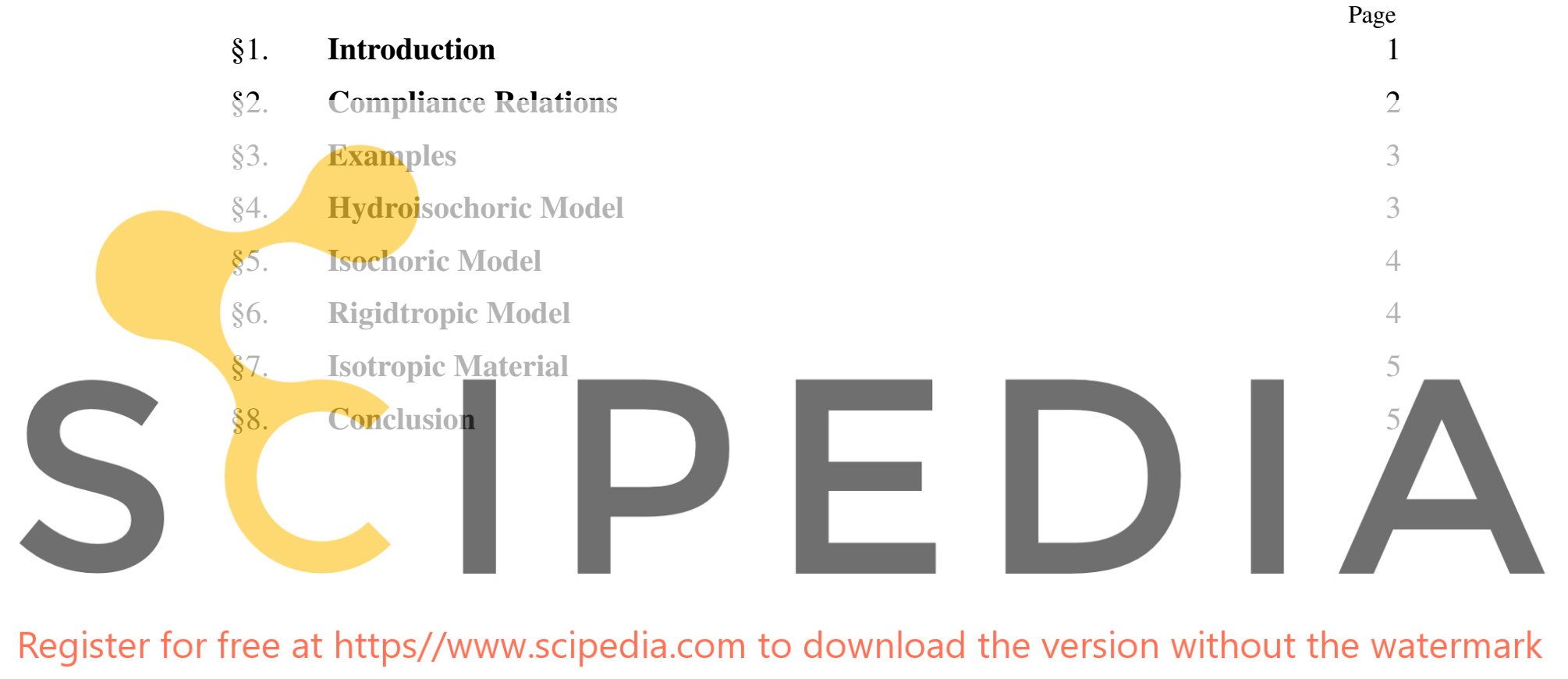




\title{
Volumetric Constraint Models for Anisotropic Elastic Solids
}

\author{
Carlos A. Felippa* and Eugenio OÑate ${ }^{\dagger}$ \\ * Department of Aerospace Engineering Sciences \\ and Center for Aerospace Structures \\ University of Colorado \\ Boulder, Colorado 80309-0429, USA
}

$\dagger$ International Center for Numerical Methods in Engineering (CIMNE)

Edificio C-1, c. Gran Capitán s/n

Universidad Politécnica de Cataluña

08034 Barcelona, Spain

\section{Summary}

We study three "incompressibility flavors" of linearly-elastic anisotropic solids that exhibit volumetric constraints:

isochoric, hydroisochoric and rigidtropic. An isochoric material deforms without volume change under any stress

system. An hydroisochoric material does se under hydrostatic stres

deformations under a certain stress pattern. Whereas the three models

differences appear for

conditions may be ham

materials of arbitrary anisotropy
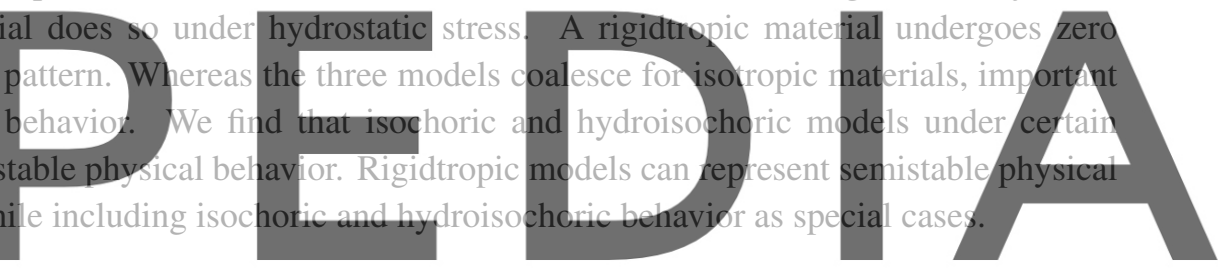

Keywords: linear elasticity, solịd, anișotropy, isotropy, rigidtropy, incompressibility, isochoric, hydroisochoric,

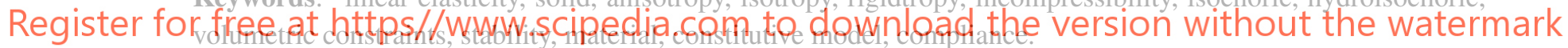

\section{Introduction}

An incompressible linearly-elastic isotropic solid does not deform under hydrostatic stress. It does not change volume under pressure. Since deviatoric and volumetric deformations uncouple, no volume change occurs under any stress state. The three volumetric constraints just stated coalesce, and it is sufficient to qualify the material as incompressible.

A more careful study is necessary for anisotropic materials. In the present Note we examine three volumetric constraint models of a linearly elastic anisotropic solid. The following definitions are used for that examination.

A material is called rigidtropic if it does not deform (i.e., experiences zero strains) under a specific stress pattern, which is a null eigenvector of the strain-stress (compliance) matrix. The term "rigidtropic" is used in the sense of "rigidity in a certain way" as defined by that eigenvector.

A material is called isochoric if it does not change volume under any applied stress system [1, Sec. 77]. Alternatively: the volumetric strain is zero under any stress state.

A material is called hydroisochoric if it is isochoric under hydrostatic stress. Isochoric materials are hydroisochoric but the converse is not necessarily true. 
As noted the three models coalesce for an isotropic material. For an arbitrary anisotropic solid, however, it will be shown that imposing a isochoric or hydroisochoric constraint may produce a compliance matrix that has at least one negative eigenvalue. This means that under some stress system the material is able to create energy, contradicting the laws of thermodynamics. Such model cannot represent a physically stable material. On the other hand, for rigidtropic behavior it is easier to control material stability for any type of anisotropy because constraints are posed directly on the spectral form.

\section{Compliance Relations}

We consider a linearly-elastic anisotropic solid in three dimensions referred to axes $\left\{x_{i}\right\}$. Stresses $\sigma_{i j}$ and strains $e_{i j}$ will be arranged as 6-component column vectors constructed from the respective tensors through the usual conventions of structural mechanics:

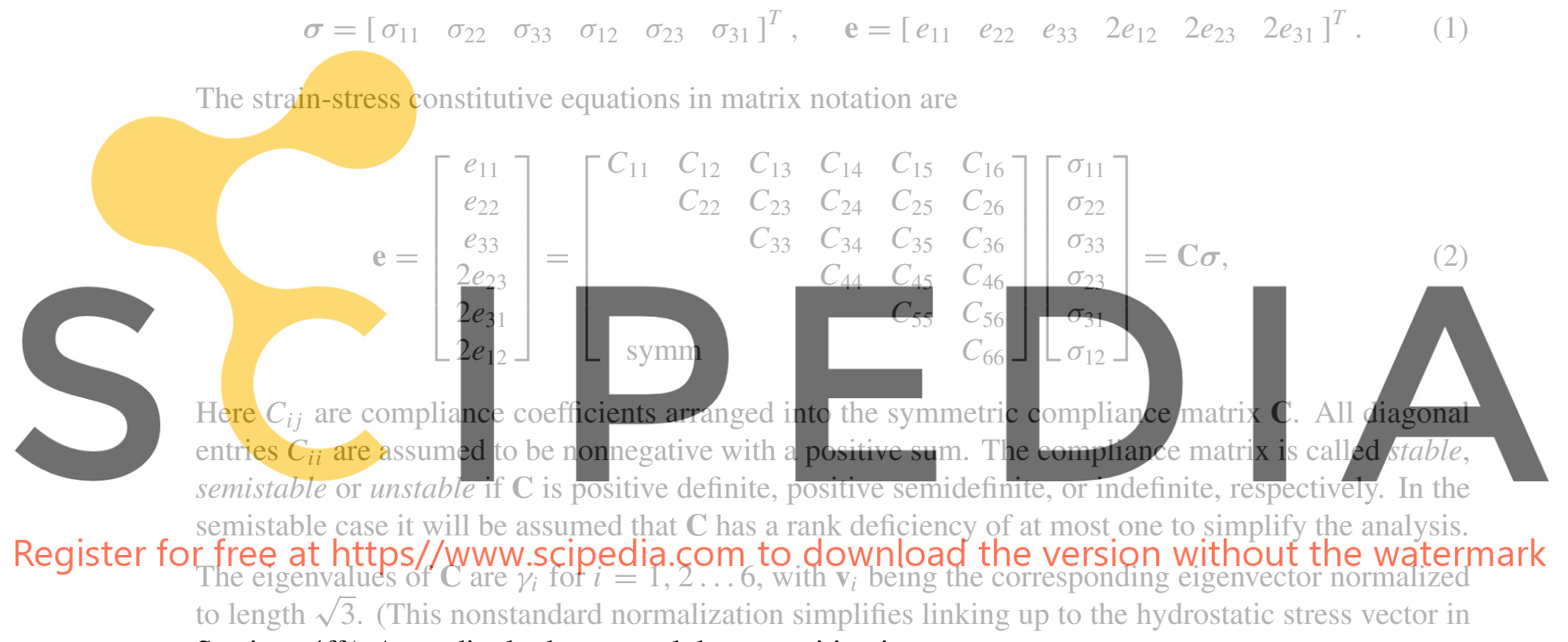

Sections 4ff.) Accordingly the spectral decomposition is

$$
\mathbf{C}=\frac{1}{3} \sum_{i=1}^{6} \gamma_{i} \mathbf{v}_{i} \mathbf{v}_{i}^{T}, \quad \mathbf{v}_{i}^{T} \mathbf{v}_{j}=3 \delta_{i j},
$$

where $\delta_{i j}$ is the Kronecker delta. The eigenvalues will be arranged so that $\gamma_{1}=\gamma_{\min }$ is the algebraically smallest one and $\gamma_{6}=\gamma_{\max }$ the maximum. For stable or semistable models, $\gamma_{1} \geq 0$ and $\gamma_{j}>0$ for $j=2, \ldots 6$.

If $\gamma_{1}=0$ the material is rigidtropic according to the definition given in the Introduction, with $\mathbf{v}_{1}$ defining the corresponding stress pattern. The volumetric strain is $e_{v}=e_{11}+e_{22}+e_{33}$. Isochoric behavior is mathematically characterized by $e_{v}=0$ under any $\sigma$. Hydroisochoric behavior means that $e_{v}=0$ under $\sigma_{p}=p\left[\begin{array}{llllll}1 & 1 & 1 & 0 & 0 & 0\end{array}\right]^{T}$ for any $p$. These constraints are mathematically expressed in terms of $\mathbf{C}$ as follows.

Rigidtropic: $\quad \gamma_{1}=0, \gamma_{i}>0, i=2, \ldots 6$.

hydroisochoric: $\quad C_{11}+C_{22}+C_{33}+2 C_{12}+2 C_{13}+2 C_{23}=0$.

Isochoric: $C_{1 j}+C_{2 j}+C_{3 j}=0, j=1,2,3$. 
Diagonal compliances are often known reliably from extensional and torsion tests. Off diagonal entries are typically less amenable to accurate measurement. Volumetric constraints, for example on volume change, are checked with triaxial tests. In any case, such constraints may be satisfied only approximately. Reference [2] discusses projection and scaling techniques for finding a "reference model" that satisfies constraints accurately while removing spurious instabilities due to experimental noise.

\section{Examples}

The following examples of compliance matrices pertain to an orthotropic material with the $\left\{x_{i}\right\}$ aligned with the principal material axes. The diagonal entries are kept the same. The three nonzero off-diagonal entries are adjusted to meet the definitions (4).

\section{Rigidtropic:}
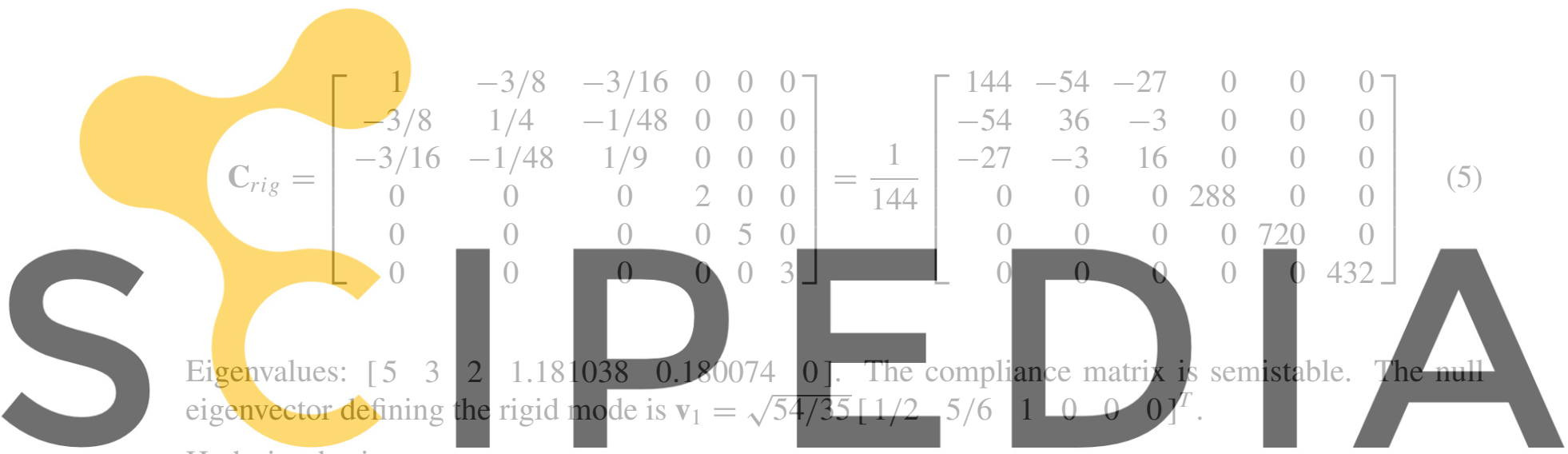

Hydroisochoric:

Register for free at https//www.scipedia.com to download the version without the watermark

$\mathbf{C}_{\text {hyd }}=\left[\begin{array}{ccccccc}1 & -11 / 27 & -95 / 432 & 0 & 0 & 0 \\ -11 / 27 & 1 / 4 & -23 / 432 & 0 & 0 & 0 \\ -95 / 432 & -23 / 432 & 1 / 9 & 0 & 0 & 0 \\ 0 & 0 & 0 & 2 & 0 & 0 \\ 0 & 0 & 0 & 0 & 5 & 0 \\ 0 & 0 & 0 & 0 & 0 & 3\end{array}\right]=\frac{1}{432}\left[\begin{array}{rrrrrr}432 & -176 & -95 & 0 & 0 & 0 \\ -176 & 108 & -23 & 0 & 0 & 0 \\ -95 & -23 & 48 & 0 & 0 & 0 \\ 0 & 0 & 0 & 576 & 0 & 0 \\ 0 & 0 & 0 & 0 & 1440 & 0 \\ 0 & 0 & 0 & 0 & 0 & 864\end{array}\right]$

Eigenvalues: $\left[\begin{array}{lllllll}5 & 3 & 2 & 1.208689 & 0.211580 & -0.059158\end{array}\right]$. The compliance matrix is unstable.

Isochoric:

$$
\mathbf{C}_{i s o}=\left[\begin{array}{cccccc}
1 & -41 / 72 & -31 / 72 & 0 & 0 & 0 \\
-41 / 72 & 1 / 4 & 23 / 72 & 0 & 0 & 0 \\
-31 / 72 & 23 / 72 & 1 / 9 & 0 & 0 & 0 \\
0 & 0 & 0 & 2 & 0 & 0 \\
0 & 0 & 0 & 0 & 5 & 0 \\
0 & 0 & 0 & 0 & 0 & 3
\end{array}\right]=\frac{1}{144}\left[\begin{array}{rrrrrr}
144 & -82 & -62 & 0 & 0 & 0 \\
-82 & 36 & 46 & 0 & 0 & 0 \\
-62 & 46 & 16 & 0 & 0 & 0 \\
0 & 0 & 0 & 288 & 0 & 0 \\
0 & 0 & 0 & 0 & 720 & 0 \\
0 & 0 & 0 & 0 & 0 & 432
\end{array}\right]
$$

Eigenvalues: $\left[\begin{array}{llllll}5 & 3 & 2 & 1.508781 & 0 & -0.147669\end{array}\right]$. The compliance matrix is unstable. 


\section{Hydroisochoric Model}

Assume that the material modeled by (2) is hydroisochoric. Consequently

$$
\mathbf{C} \sigma_{p}=\left[\begin{array}{cccccc}
C_{11} & C_{12} & C_{13} & C_{14} & C_{15} & C_{16} \\
& C_{22} & C_{23} & C_{24} & C_{25} & C_{26} \\
& & C_{33} & C_{34} & C_{35} & C_{36} \\
& & & C_{44} & C_{45} & C_{46} \\
& & & & C_{55} & C_{56} \\
\text { symm } & & & & C_{66}
\end{array}\right]\left[\begin{array}{c}
p \\
p \\
p \\
0 \\
0 \\
0
\end{array}\right]=\left[\begin{array}{c}
p\left(C_{11}+C_{12}+C_{13}\right) \\
p\left(C_{12}+C_{22}+C_{23}\right) \\
p\left(C_{13}+C_{23}+C_{33}\right) \\
2 e_{12} \\
2 e_{23} \\
2 e_{31}
\end{array}\right]=\left[\begin{array}{c}
e_{11} \\
e_{22} \\
e_{33} \\
2 e_{23} \\
2 e_{31} \\
2 e_{12}
\end{array}\right],
$$

with $e_{v}=e_{11}+e_{22}+e_{33}=p\left(C_{11}+C_{22}+C_{33}+2 C_{12}+2 C_{13}+2 C_{23}\right)=0$.

(The value of the shear strains is of no interest.) The complementary energy density produced by $\sigma_{p}$ is

$$
\mathcal{U}_{p}^{*}=\frac{1}{2} \sigma_{p}^{T} \mathrm{C} \sigma_{p}=\frac{1}{2} p\left(e_{11}+e_{22}+e_{33}\right)=\frac{1}{2} p e_{v}=0 .
$$

But $\gamma_{p}=\mathcal{U}_{p}^{*} /\left(\sigma_{p}^{T} \sigma\right)=\mathcal{U}_{p}^{*} /\left(3 p^{2}\right)=0$ is the Rayleigh quotient of $\sigma_{p}$ with $\mathbf{C}$. According to the Courant-Fisher theorem [2], $\gamma_{p}$ must lie in the closed interval $\left[\gamma_{\min }, \gamma_{\max }\right]$ :

$$
\gamma_{1} \leq \gamma_{p}=0 \leq \gamma_{6}
$$

If $\sigma_{p}$ is not an eigenvector of $\mathbf{C}: \mathbf{C} \sigma_{p} \neq \mathbf{0}$, the leftmost equality in (10) is not possible. Consequently
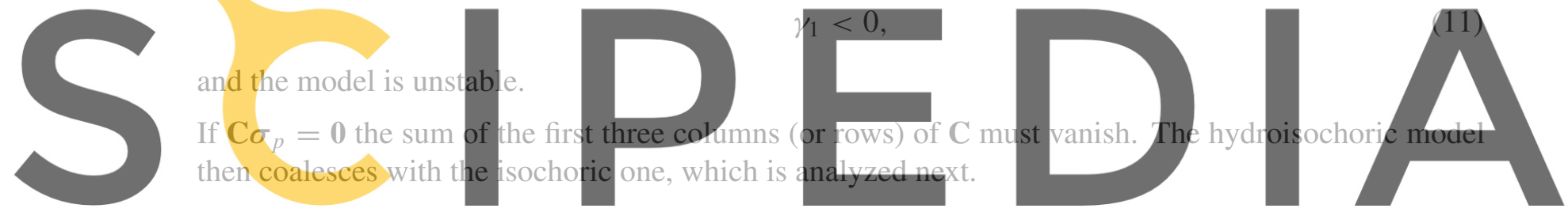

\section{Isochoric Model}

Register for free at https. / www ssipedia, fom to download the version withoyt the watermark

$\sigma_{p}$ is a null eigenvector of $\mathbf{C}$. The Rayleigh quotient test (10) does not offer sufficient information on stability and a deeper look at $\mathbf{C}$ is required. Nonetheless a sufficient criterion for instability can be derived by considering the upper $3 \times 3$ principal minor $\tilde{\mathbf{C}}$. From the last of (4), $\tilde{\mathbf{C}}$ must have the form:

$$
\tilde{\mathbf{C}}=\left[\begin{array}{ccc}
C_{11} & C_{12} & C_{13} \\
& C_{22} & C_{23} \\
\text { symm } & C_{33}
\end{array}\right]=\left[\begin{array}{ccc}
C_{11} & \frac{1}{2}\left(C_{33}-C_{11}-C_{22}\right) & \frac{1}{2}\left(C_{22}-C_{11}-C_{33}\right) \\
& C_{22} & \frac{1}{2}\left(C_{11}-C_{22}-C_{33}\right) \\
\text { symm } & C_{33}
\end{array}\right] .
$$

This matrix is singular. Taking $\alpha=C_{11} / C_{22}$ and $\beta=C_{11} / C_{33}$ for convenience, an eigenvalue analysis shows that $\tilde{\mathbf{C}}$ is indefinite if

$$
2\left(\frac{1}{\alpha}+\frac{1}{\beta}\right)<1+\left(\frac{1}{\alpha}-\frac{1}{\beta}\right)^{2}
$$

and is positive semidefinite if the inequality is reversed. If $\tilde{\mathbf{C}}$ is indefinite, so is $\mathbf{C}$ and the model is unstable. If $\tilde{\mathbf{C}}$ is semidefinite, an eigenvalue analysis of the complete $\mathbf{C}$ is required to decide on stability. The stability regions of $\tilde{\mathbf{C}}$ are shown in Figure 1, where "potentially semistable" indicates that confirmation by a analysis of the full $\mathbf{C}$ is required. An exception is an orthotropic material referred to principal material axes, in which case no further tests are necessary if $C_{44}, C_{55}$ and $C_{66}$ are positive.

Figure 1 illustrates that a wide range of diagonal compliances in $\tilde{\mathbf{C}}$ is detrimental to stability. For example if $\alpha=\beta$, instability is guaranteed to happen for $\alpha>4$. 


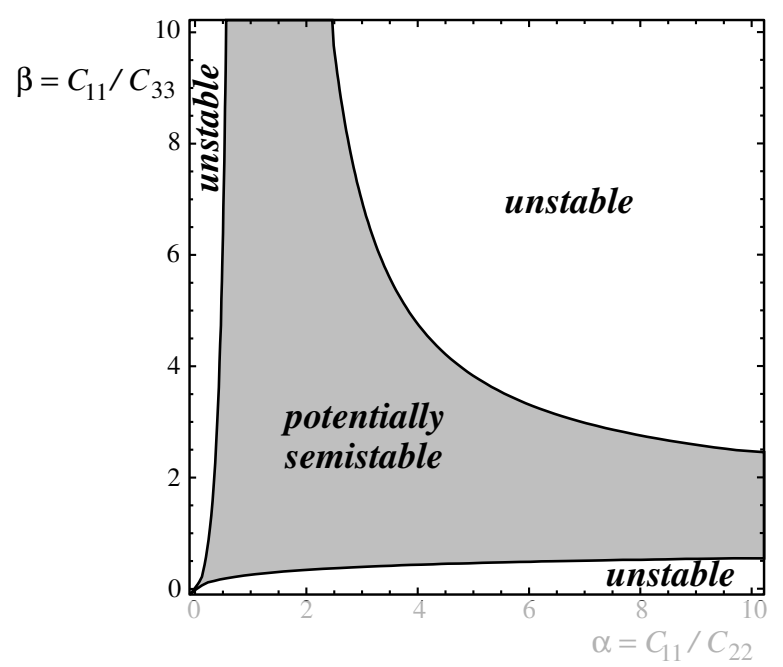

Figure 1. Stability chart for the principal minor (12) of an isochoric material as function of the ratios $C_{11} / C_{22}$ and $C_{11} / C_{33}$.

\section{Rigidtropic Model}

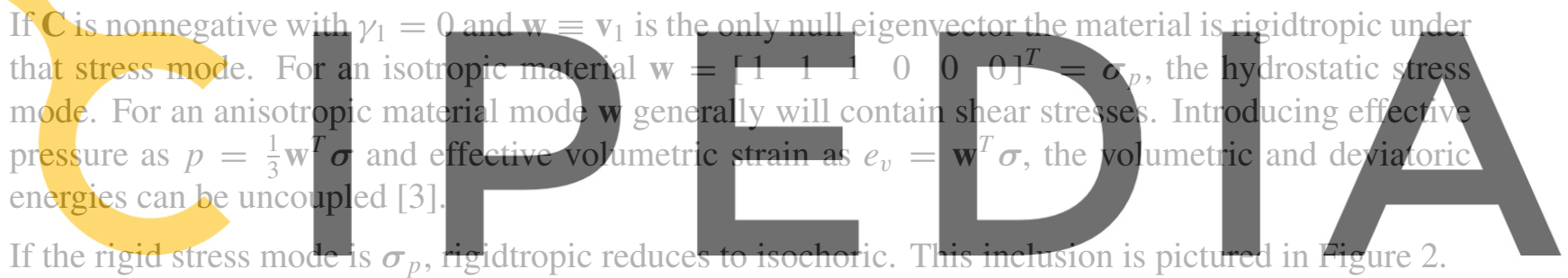

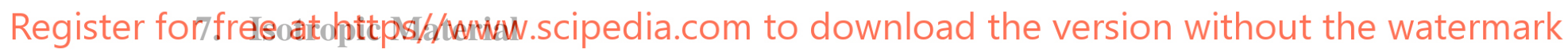

If the solid is isotropic with elastic modulus $E>0$ and Poisson's ratio $v$,

$$
\mathbf{C}=\frac{1}{E}\left[\begin{array}{cccccc}
1 & -v & -v & 0 & 0 & 0 \\
& 1 & -v & 0 & 0 & 0 \\
& 1 & 0 & 0 & 0 \\
& & 2(1+v) & 0 & 0 \\
& & & 2(1+v) & 0 \\
\text { symm } & & & & 2(1+v)
\end{array}\right]
$$

Under hydrostatic stress $\sigma_{p}, e_{v}=3(1-2 v) p / E$, which vanishes for $v=\frac{1}{2}$. It is easy to verify that if $v=\frac{1}{2}, e_{v}=0$ for any $\sigma$ and the material is isochoric. Furthermore $\sigma_{p}$ is the only null eigenvector of $\mathbf{C}$. Consequently $\gamma_{p}=\gamma_{1}=0$ and $\mathbf{C}$ has no negative eigenvalues. The definitions of rigidtropic, incompressible and isochoric behavior coalesce for this model.

\section{Conclusion}

It remains to pin down the label "incompressible." In continuum mechanics this term means that the stress is determined by the deformation history only up to a hydrostatic pressure or "extra stress" $p$ [4, Sec. 30]. This is equivalent to what we call here the hydroisochoric model, which as previously 


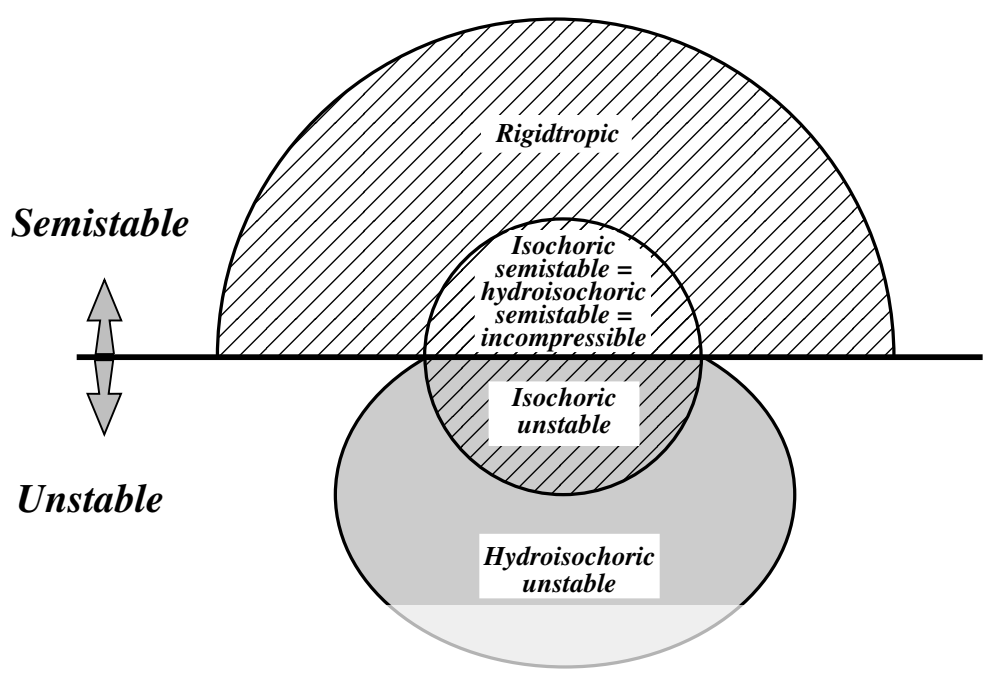

Figure 2. Schematic of inclusions between rigidtropic, isochoric and hydroisochoric models. The crosshatched area marks a singular $\mathbf{C}$ matrix.

shown for semistable materials merges with the isochoric model. Restricting attention to the semistable case, the model nesting is:

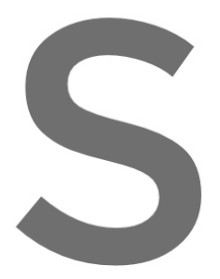
(n) $D$ behavior, and no special distinction for the incompressible case needs to be made.

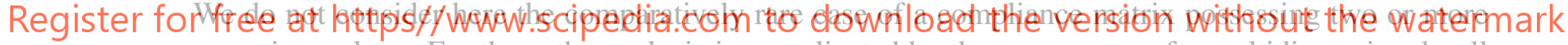 zero eigenvalues. For those the analysis is complicated by the appearance of a multidimensional null space. Such "multi-rigidtropic" models require separate treatment.}

\section{Acknowledgements}

The work of the first author has been supported by the Spanish Ministerio of Educación y Cultura through a faculty fellowship while visiting CIMNE over the period April through June 2002.

\section{References}

[1] C. Truesdell and R. Toupin, The classical field theories, in Handbook der Physik, ed. by S. Flugge, Vol III/1, Springer-Verlag, Berlin, 1960.

[2] R. Courant and D. Hilbert, Methods of Mathematical Physics, Vol I, Interscience, New York, 1953.

[3] C. A. Felippa and E. Oñate, Stress, strain and energy splittings for anisotropic elastic solids under volumetric constraints, CIMNE Report No. 217, International Center for Numerical Methods in Engineering, Barcelona, Spain, July 2002. Submitted to Computers and Structures

[4] C. Truesdell and W. Noll, The nonlinear field theories of mechanics, in Handbook der Physik, ed. by S. Flugge, Vol III/3, Springer-Verlag, Berlin, 1965. 